\title{
Impact of Bariatric Surgery on Ghrelin and Obestatin Levels in Obesity or Type 2 Diabetes Mellitus Rat Model
}

\author{
Donglei Zhou, ${ }^{1}$ Xun Jiang, ${ }^{1}$ Weixing Ding, ${ }^{1}$ Dingyu Zhang, Lei Yang, \\ Chengzhu Zhen, ${ }^{3}$ and Liesheng $\mathrm{Lu}^{1}$ \\ ${ }^{1}$ Department of General Surgery, Shanghai Tenth People's Hospital of Tongji University, Shanghai 200072, China \\ ${ }^{2}$ August First Physical Culture and Sports Team, Haidian District, Beijing 100091, China \\ ${ }^{3}$ Department of General Surgery, Changhai Hospital of the Second Military Medical University, Shanghai 200433, China \\ Correspondence should be addressed to Liesheng Lu; lushengliell@hotmail.com
}

Received 21 June 2013; Revised 21 November 2013; Accepted 21 November 2013; Published 10 February 2014

Academic Editor: Norman Cameron

Copyright (C) 2014 Donglei Zhou et al. This is an open access article distributed under the Creative Commons Attribution License, which permits unrestricted use, distribution, and reproduction in any medium, provided the original work is properly cited.

\begin{abstract}
We aimed to evaluate the therapeutic efficacy on weight control by different bariatric surgeries and investigate the ghrelin and obestatin changes after these surgeries in obesity and nonobese type 2 diabetes mellitus (T2DM) rats. Obese rats were randomly assigned to receive sleeve gastrectomy ( $\mathrm{SG}, n=8$ ), minigastric bypass (MGBP, $n=8$ ), roux-en-Y gastric bypass (RYGBP, $n=8$ ), and sham operation $(\mathrm{SO}, n=4)$. Another 4 rats served as control. Besides, Goto-Kakisaki $(\mathrm{GK})$ rats were also randomly divided into similar groups except for total gastrectomy (TG, $n=8$ ) group. The results showed that in obese rats, weigh loss in RYGBP group was similar to that in MGBP group but larger than that in SG group. Ghrelin significantly increased in RYGB group, but obestatin increased in MGBP group. Ghrelin/obestatin ratio significantly decreased in SG group. In GK rats, weight loss was most obvious in TG group. Postoperatively, ghrelin was significantly increased in MGBP and RYGB groups but decreased in TG group. Obestatin also showed an increase in MGBP and RYGB groups. Ghrelin/obestatin in TG group decreased significantly. In conclusion, RYGB and MGBP may be more suitable for obese rats, but TG may be the best strategy for T2DM rats to control weight with different mechanisms.
\end{abstract}

\section{Introduction}

Obesity and type 2 diabetes mellitus (T2DM) have currently become 2 of the most common chronic diseases globally [1]. According to World Health Organization estimates, the numbers of people with obesity and T2DM worldwide will reach 700 million and 300 million, respectively, by 2015 [2]. Both diseases are closely associated with excessive food intake and a sedentary lifestyle. Thus, lifestyle modification (including diet, physical activity, and behavior therapy) is usually suggested to promote weight loss and prevent the disease development [3]. However, none of these treatments has long-term success because it is difficult to maintain healthy eating and exercise behaviors over time [4].

Recently, accumulating evidence has demonstrated that bariatric surgery is a more effective way to produce greater and sustained body weight reduction (weight loss of $50 \%$ to $75 \%$ of excess body weight) compared with nonsurgical treatments $[5,6]$. The commonly used bariatric surgeries include roux-en-Y gastric bypass (RYGB), minigastric bypass (MGB) and sleeve gastrectomy (SG). Lee et al. reported that participants assigned to RYGB lost more weight than the SG group [7]. In addition, they also found that MGBP was superior to RYGB in lowering the body mass index (BMI) and increasing the excess weight loss $[8,9]$. These findings indicate that the effectiveness sequence was MGB > RYGB > SG. However, Padwal et al. obtained the inconsistent conclusions: differences in BMI levels from baseline are as follows: MGB $\left[-11.3 \mathrm{~kg} / \mathrm{m}^{2}\right]$, SG $\left[-10.1 \mathrm{~kg} / \mathrm{m}^{2}\right]$, and RYGB $\left[-9.0 \mathrm{~kg} / \mathrm{m}^{2}\right][10]$. Thus, further effect study is still needed.

Furthermore, it is hypothesized that the preliminary encouraging results with these methods in weight loss may be attributed not only to the restriction of the gastric capacity, but also to the drastic changes in hormone secretion (ghrelin 
and obestatin), leading to large decreases in appetite and food intake [11]. However, there are controversial results about the hormone changes after surgery. Some studies reported a significant decrease in ghrelin and obestatin levels after surgery [12-15], while others, increase [16-18] or no change [12]. Importantly, different surgeries achieve different influence, for example, plasma obestatin concentrations were shown to be significantly increased in patients after SG but without any alteration after GB [19]. Thus, further mechanism study is still required.

The aim of this study was to further evaluate the therapeutic efficacy on weight control by different surgeries and investigate the changes of ghrelin and obestatin before and after these surgeries in obesity and non-obese T2DM rat models.

\section{Materials and Methods}

2.1. Experimental Animals and Grouping. All animal studies have been approved by China Ethics Committee and performed in accordance with the ethical standards. A total of 70 specific pathogen-free (SPF) grade male Sprague-Dawley (SD) rats aging 6 weeks old with an average weight of $210 \pm$ $20 \mathrm{~g}$ and 40 SPF grade male Goto-Kakisaki (GK) rats (a non-obese model of spontaneous T2DM) aging 9 weeks old with an average weight of $340 \pm 20 \mathrm{~g}$ were purchased from Shanghai Slyke Laboratory Animal Corp. (Certificate no.: SCXK 2008-0003). All animals were weighed and bred to adapt to the environment for 1 week. Then SD rats were randomly divided into 2 groups to be fed with a normal diet $(n=35)$ and high-lipid diet $(n=35)$ for 7 weeks. If the weight of rats in obese group exceeded $20 \%$ of average weight of rats in normal group, animal model of obesity was considered to be established successfully. Subsequently, obese rats were randomly assigned to receive SG $(n=8), \operatorname{MGBP}(n=8)$, YGBP $(n=8)$, and sham operation (SO, $n=4)$. Four rats served as control without any treatment $(\mathrm{NC}, n=4)$. Another 6 rats were used as supplements in case of an unexpected death in surgery. GK rats were also fed with a high-fat diet for 10 weeks and then randomly divided into SG group $(n=8)$, MGBP $(n=8)$, YGBP $(n=8)$, total gastrectomy (TG) group $(n=8)$, SO group $(n=4)$, and NC group $(n=4)$. All procedures were conducted according to the Guide for the Care and Use of Laboratory Animals.

2.2. Surgical Procedures. The rats were fasted for $7 \mathrm{~h}$ preoperatively for upper gastrointestinal surgery, but $24 \mathrm{~h}$ for lower gastrointestinal surgery. Glucose can be added in animal's drinking water to supplement energy and increase their tolerance for anesthesia. The rats were anesthetized with $1 \%$ sodium pentobarbital solution $(30-50 \mathrm{mg} / \mathrm{kg}$ ) injected intraperitoneally into rats for $60-120 \mathrm{~min}$. Antibiotic prophylaxis was applied with intramuscular 5\% cefradine $(0.02 \mathrm{~g} / \mathrm{kg})$. Sleeve gastrectomy (SG) involves the creation of a reduced stomach lumen with a 48 -Fr bougie at the lesser curvature through the removal of gastric tissues along the greater curvature from the fundus to the antrum [20]. In MGBP surgery, a long gastric tube was created approximately
$1.5 \mathrm{~cm}$ to the left of the lesser curvature from the antrum to the angle of His. A loop gastroenterostomy was created with the small bowel about $20 \mathrm{~cm}$ distal to the ligament of Trietz [8]. In YGBP group, the dissection began directly on the lesser curvature of the stomach, and a gastric pouch of about $20 \%$ of the total gastric volume was created. The pouch was anastomosed to a Roux limb of jejunum created by division of the jejunum $15 \mathrm{~cm}$ distal to the ligament of Treitz and anastomosing the afferent biliopancreatic limb to the jejunum $15 \mathrm{~cm}$ distally [21]. A total gastrectomy (TG) was performed with the removal of the entire stomach and end-to-end anastomosis of the esophagus and duodenum. Sham operated (SO) controls received manipulation of the stomach and a transverse enterotomy at the same position of the proximal jejunum; however, this was reclosed without forming an anastomosis. All surgeries were performed with the first author. The surgery time was 40-60 min for SG and TG, but 60-90 min for MGBP and YGBP. Postoperatively, animals were given ad libitum access to water and a highfat diet. Additional doses of fluid infusion (glucose) were also administered to accelerate recovery.

2.3. Body Weight Measurement. Electronic scale was used to record body weight of rats every day under their quiet and awake condition until 12 weeks or 20 weeks postoperatively for obese rats, but 5 weeks postoperatively for GK rats.

2.4. Ghrelin and Obestatin Measurement. Venous blood from angular vein was collected into potassium/EDTA-coated tube containing $0.6 \mathrm{TIU} / \mathrm{mL}$ aprotinin. Samples were then centrifuged at $1600 \mathrm{~g}$ for $15 \mathrm{~min}$ at $4^{\circ} \mathrm{C}$ and plasma kept at $-80^{\circ} \mathrm{C}$ for later analyses. Fasting blood glucose was determined by using spectrophotometer. Ghrelin and obestatin plasma levels before, 4 weeks, and 12 weeks after operation in obesity rats and before, 2 weeks, and 5 weeks after operation in GK rats were determined by radioimmunoassay (RIA, Phoenix Pharmaceuticals, Inc., USA) according to the manual descriptions.

2.5. Statistical Analysis. All data were analyzed by SPSS 13.0 software and were displayed by mean \pm SD. Paired Student's $t$ test was used to compare data before and after the operation, Student's $t$-test or $t^{\prime}$ test was calculated according to the homogeneity of variance detected by $F$ test, and analysis of covariance (ANCOVA) further corrected the differences among groups before operation. Pearson's correlation coefficient was used to test the correlation among the indicators. $P<0.05$ was considered statistically significant.

\section{Results}

3.1. Obesity and T2DM Rat Models. After being fed with a high-fat diet for 3 weeks, body weight of obesity rats group was significantly higher than that of the control group. In the 7 th week, obesity rat model had been successfully established (Figure 1(a)). By dissection, we also found that body fat content including subcutaneous and intra-abdominal fat in obesity group was significantly higher than that in the control. 


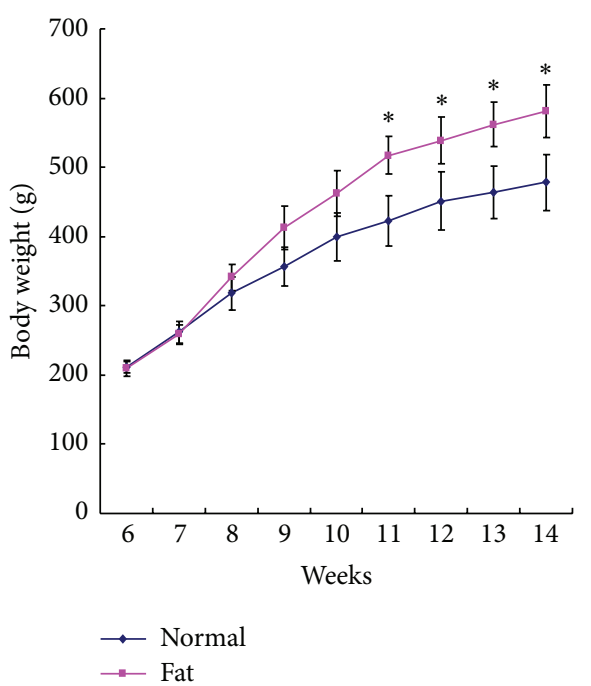

(a)

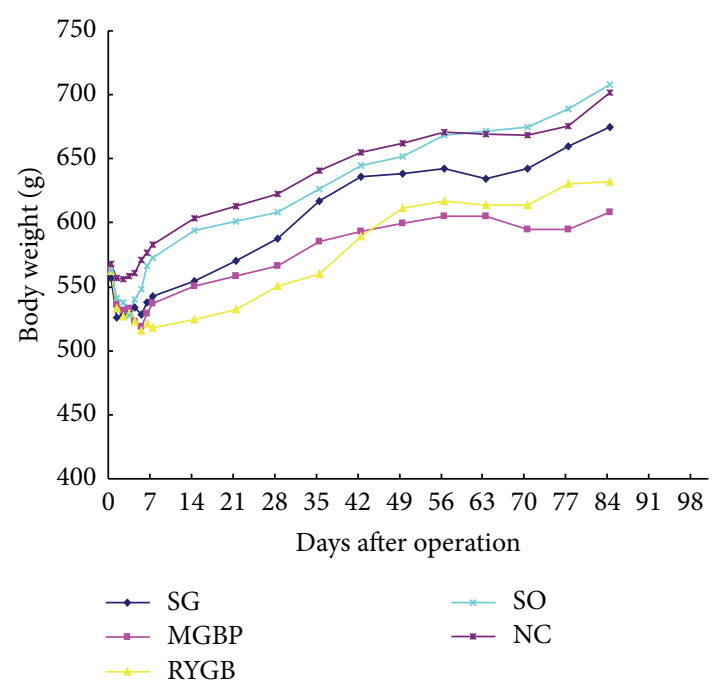

(c)

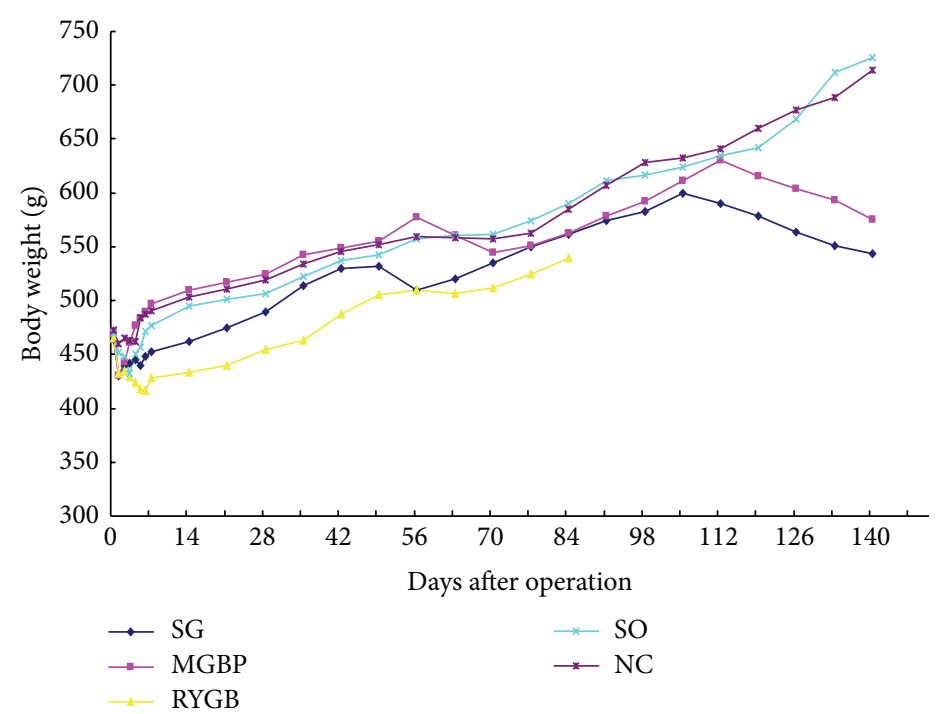

(b)

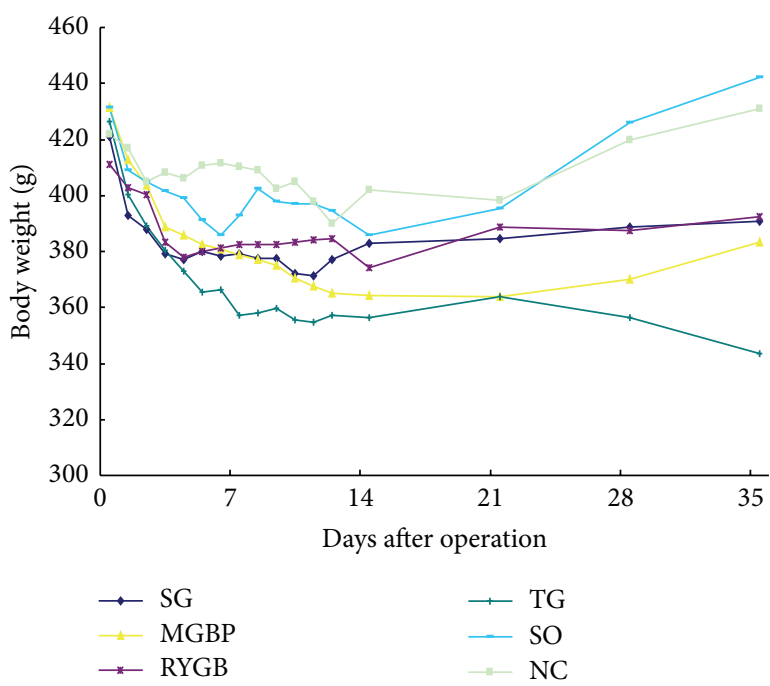

(d)

FIGURE 1: Body weight changes after different diets feed. (a) Preoperative body weights of rats with a normal diet or high-lipid diet; (b) postoperative body weight in normal rats; (c) postoperative body weight in obese rats; (d) postoperative body weight in Goto-Kakisaki (GK) rats. ${ }^{*}$ Compared with the body weight of rats before experiment. SG, sleeve gastrectomy; MGBP, minigastric bypass; RYGBP, roux-en-Y gastric bypass; SO, sham operation; NC, normal control; TG, total gastrectomy.

The percentage for testosterone and perinephric fat in body weight of obese rats was $>10 \%$, while the percentage in normal rats was only $<5 \%$. The average level of serum triglyceride and total cholesterol in obesity group was more than $50 \%$ but $25 \%$ of normal group, respectively. After being fed with highfat diet for 10 weeks, fasting blood glucose was significantly higher in GK rats than that in the normal control $(23.6 \pm$ $4.9 \mathrm{mmol} / \mathrm{L}$ versus $5.6 \pm 1.46 \mathrm{mmol} / \mathrm{L}, P<0.001)$.

\subsection{Body Weight Changes of Rats after Different Surgeries.} There was no difference in body weight among the groups before operation in normal rats. Weight loss existed in all the groups within 1 week after operation, of which weight loss in RYGB group was the greatest $(P<0.01)$. One week later, weight showed a gradual increase except that in RYGB group and MGBP group, and weight in RYGB group was significantly lower than MGBP group $(P<0.05)$. In 11th week after operation, only weight in RYGB group was still lower than the other groups $(P<0.05)$. In $15-16$ th week after operation, weight in SO and NC groups increased with the age growing, while weight in MGBP and SG groups began to decrease and was significantly lower than the other groups in 20th week after operation $(P<0.01)$ (Figure 1(b)).

In the obese rats group, there was also no difference in weight among the groups before operation. In 2 nd week after operation, the average weight in RYBG, SG, and MGBP groups was significantly lower than that in the $\mathrm{NC}$ and SO 
groups, and weight loss in RYBG group was the greatest $(P<$ $0.01)$. In 6 th week after operation, weight in SG group was similar to that in NC group but decreased in 9th week after operation. In 12th week after operation, weight in RYBG and MGBP groups was significantly lower than that in NC group $(P<0.01)$, but there was no significant change in weight between SG group and NC group $(P>0.05$, Figure $1(c))$.

In GK rats, there was no difference in body weight among the groups before operation. Weight loss varied in all the groups within 1 week after operation. One week later, weight increased in $\mathrm{NC}$ and SO groups but continued to fall in the operation groups. The weight was NC, SO > SG, RYG, MGBP $>$ TG $(P<0.01)$ on the 10th day after operation, NC, SO > SG, RYGB > TG, MGBP $(P<0.05)$ in the 2nd week after operation, and NC, SO > SG, RYGB, MGBP $(P<0.01)$, SG, RYGB, MGBP $>$ TG $(P<0.01)$ in the 5 th week after operation (Figure 1(d)).

\subsection{Changes of Ghrelin, Obestatin Plasma Levels and the} Ratio of Ghrelin/Obestatin. In normal rats before operation, ghrelin levels were significantly lower in RYGB group than those in NC group $(P=0.021)$, but no differences in obestatin levels and the ratios of ghrelin/obestatin were observed among all the groups. After operation, ghrelin levels in RYGB and MGBP groups significantly increased, but ghrelin levels in SG group only showed a significant increase in 12 th week after operation. Obestatin level increased at different degrees in different groups after operation, but no statistical significance was present among groups when growth factors were eliminated through ANCOVA. Ratio of ghrelin/obestatin decreased in SO group $(P<0.05)$ after operation and in NC group 4 weeks after operation $(P<$ $0.01)$. There was no significant difference when the data were corrected by ANCOVA (Figure 2(a)).

In obesity rats group, ghrelin, obestatin, and ghrelin/obestatin showed no difference among all the groups before operation but were higher than those in normal rats. Ghrelin levels significantly increased in RYGB group postoperatively $(P<0.05)$ and increased in SG group 4 weeks after operation $(P<0.01)$. Obestatin levels only significantly increased after operation in MGBP group $(P<$ $0.01)$. Ghrelin/obestatin ratio decreased in SG group 12 weeks after operation $(P<0.05)$ and in NC group 4 weeks after operation $(P<0.01)$ compared with that before operation (Figure 2(b)).

In GK rats before operation, ghrelin plasma level in MGBP group was lower than that in NC group, while there was no difference among other groups. At 2 weeks postoperatively, ghrelin plasma level in all the groups except TG group was increased at different degree. At 5 weeks postoperatively, ghrelin plasma level in MGBP and RYGB groups was higher than that before operation. Ghrelin plasma level in TG group was significantly lower than the other groups and that before operation. In GK rats before operation, obestatin in MGBP group was lower than that in NC group $(P=0.033)$, while obestatin in TG group was higher than that in NC group $(P=0.041)$. After operation, obestatin plasma level in MGBP and RYGB groups was higher than that before operation.
But there was no difference in obestatin levels among the groups. Preoperatively, ghrelin/obestatin ratio showed no difference among the groups. Ghrelin/obestatin ratio in TG group decreased significantly $(P<0.01)$ after operation. In 5 weeks after operation, RYGB group was higher than before operation (Figure 2(c)).

\subsection{Correlation of Ghrelin, Obestatin, and Ghrelin/Obestatin} Levels with Body Weight in Obese Rats or GK Rats. From Table 1, we can see that there was a positive relationship between ghrelin, obestatin plasma levels and body weight in normal rats, while there was a negative relationship between ghrelin/obestatin and body weight in both normal rats and obese rats. No correlation between ghrelin, obestatin plasma levels, ghrelin/obestatin, and body weight was present in GK rats.

\section{Discussion}

Our present study confirms the efficacy of bariatric surgery for the cure of obesity and T2DM rats. However, in obese rats, weigh loss in RYGBP group was similar to that in MGBP group, but larger than that in SG group. For GK rats, the obvious weight loss occurred in TG and RYGB groups. These findings suggest that participants assigned to RYGB are more likely to achieve remission of obesity and T2DM, which are in accordance with previous studies [19, 22]. Recent studies even suggest conversion from SG to RYGB when the weight regains $[23,24]$. The results indicated that weight loss was significantly improved after conversion (mean loss percentage of excess of body weight, $64.6 \%$ versus $47.1 \%$ ) and all reflux symptoms are eliminated without any medication at the end of the followup [25]. Besides, several studies also have demonstrated that body weight loss is a common sequela after total gastrectomy [26-29]. However, most studies investigated its treatment effect for patients with gastric cancer or combined with T2DM $[30,31]$. In this study we found that TG was also effective for decreasing weight for T2DM rats without gastric cancer or obesity and glucose level was still lower than that before operation when rats restored the food intake (Supplementary Figure 1 in Supplementary Materials available online at http://dx.doi.org/10.1155/2014/569435).

To reveal the mechanism of surgical treatment, roles of gastrointestinal peptide hormones such as ghrelin and obestatin raised our concerns. Ghrelin is a 28 -amino-acid, appetite-stimulating peptide hormone secreted predominantly by the food-deprived stomach in rats and humans [32]. Upregulated ghrelin expression stimulates appetite and food intake, downregulates energy expenditure, and conserves body fat, ultimately leading to weight gain [32, 33]. Besides, ghrelin suppresses insulin secretion in humans and promotes weight gain $[34,35]$. Thus, strategies that are effective for weight loss may decrease ghrelin level. However, in this study, we only observed a decrease in ghrelin level after TG, but not others, indicating the other mechanisms may be present for RYGBP, MGBP, and SG to control weight.

Obestatin is a recently discovered 23 -amino-acid peptide encoded by the ghrelin gene. Obestatin has been shown 


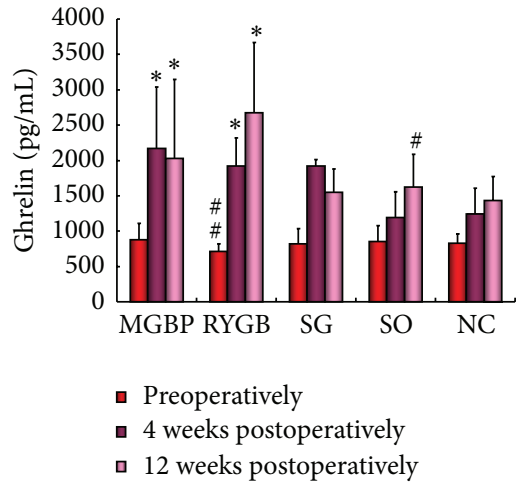

(a1)

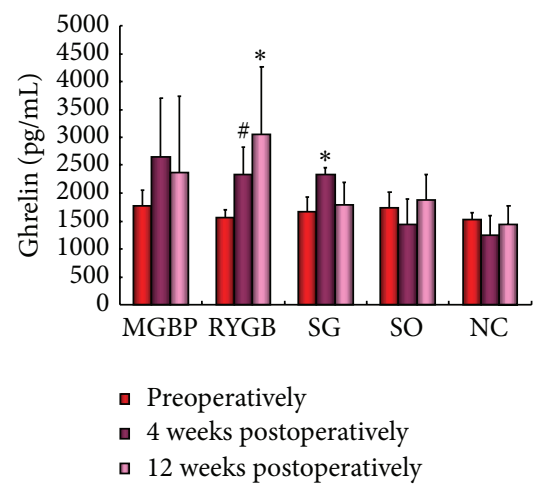

(b1)

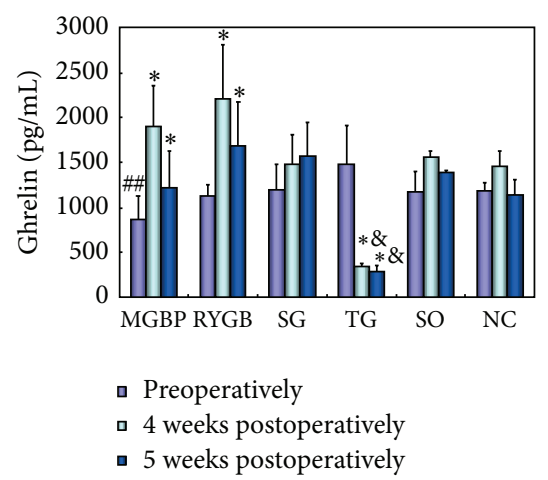

(c1)

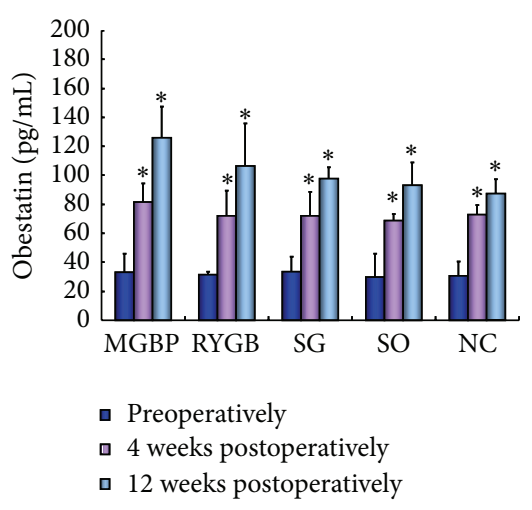

(a2)

(a)

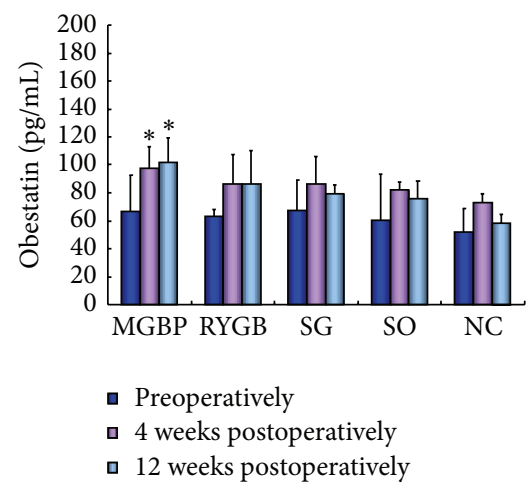

(b2)

(b)

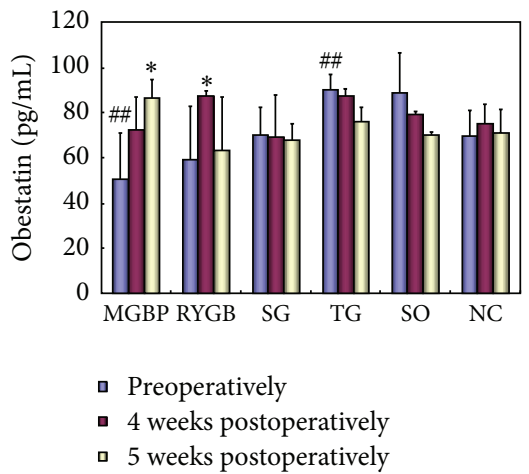

(c2)

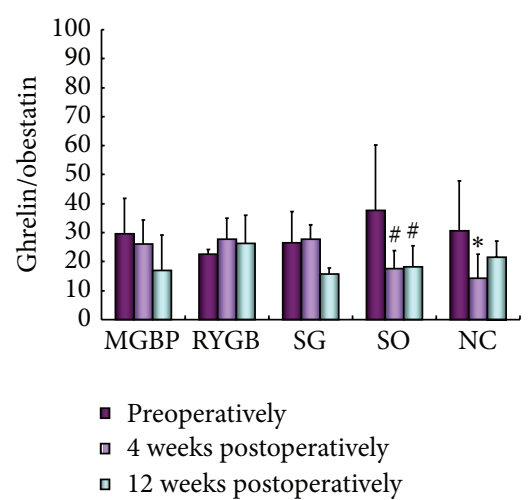

(a3)

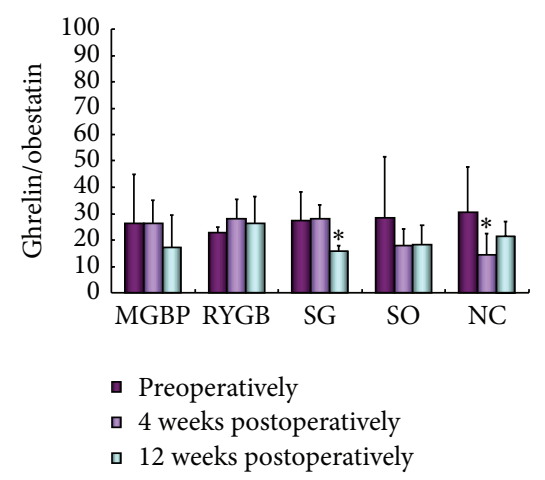

(b3)

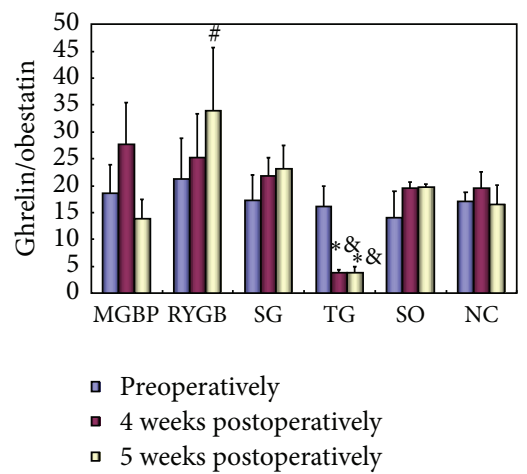

(c3)

(c)

FIGURE 2: Changes of plasma ghrelin, obestatin and ghrelin/obestatin ratio after different operation treatments. (a) Ghrelin (a1), obestatin (a2) plasma levels and the ratio of ghrelin/obestatin (a3) in normal rats; (b) ghrelin (c1) and obestatin (c2) plasma levels and ghrelin/obestatin (c3) in obese rats; (c) ghrelin (d1) and obestatin (d2) plasma levels and ghrelin/obestatin (d3) in Goto-Kakisaki (GK) rats. ${ }^{\# \#}$ Compared with NC group preoperatively, $P<0.05 .{ }^{*}$ Compared with the same group preoperatively, $P<0.05$. "Compared with the same group preoperatively, $P<0.01$. ${ }^{\&}$ Compared with other groups preoperatively, $P<0.01$. SG, sleeve gastrectomy; MGBP, minigastric bypass, RYGBP, roux-en-Y gastric bypass, $\mathrm{SO}$, sham operation; NC, normal control; TG, total gastrectomy.

to suppress food intake and decrease gastric emptying, therefore antagonizing the orexigenic effect of ghrelin [36]. As previously reported, obestatin was decreased in obese subjects and a nutritional marker reflecting body adiposity and insulin resistance [37]. Therefore, regulation of obestatin level may be another mechanism for strategies that can modulate body weight and energy homeostasis. In our study, obestatin plasma level showed no difference between obese 
TABLE 1: Correlation of ghrelin, obestatin, and ghrelin/obestatin levels with body weight.

\begin{tabular}{lcccccc}
\hline \multirow{2}{*}{ Groups } & \multicolumn{2}{c}{ Ghrelin } & \multicolumn{2}{c}{ Obestatin } & \multicolumn{2}{c}{ Ghrelin/obestatin } \\
& $r$ & $P$ & $r$ & $P$ & $r$ & $P$ \\
\hline Normal & 0.530 & 0.042 & 0.809 & $<0.001$ & -0.584 & 0.022 \\
Obesity & -0.081 & 0.773 & 0.153 & 0.587 & -0.695 & 0.004 \\
GK & 0.093 & 0.714 & -0.268 & 0.282 & 0.153 & 0.545 \\
\hline
\end{tabular}

and normal rats but was lower in GK rats. After MGBP surgery in obese rats or RYGB surgery in GK rats, obestatin was increased, suggesting that MGBP and RYGB may exert weight control role via regulation of obestatin. However, there was a study reporting that obestatin concentrations did not change after RYGB surgery in obese people [12]. Thus, ghrelin/obestatin ratio is suggested to be used to assess the treatment effect of surgery. Roth et al. reported that ghrelin/obestatin ratio was lower after surgery than before $(-14 \%, P=0.017)$ [12]. In this study, we also found that ghrelin/obestatin ratio significantly decreased in SG and TG groups. These implied that SG may play important roles for weight loss via influencing ghrelin/obestatin ratio. Our correlation analysis further addressed the evaluation function of ghrelin/obestatin ratio because only ghrelin/obestatin ratio was negatively correlated with weight loss in obese rats, but not ghrelin and obestatin.

However, there are still some limitations in this study. Although the average weight of obese rats is higher than that of age-matched normal rats, the weight is similar between the two groups at 28 weeks old (Supplementary Figure 2), suggesting that obese rats have self-limiting weight. There is a difference in food habits between human and rats, which may lead to different surgical results between rats and human being. Thus, further human studies are still needed.

\section{Conclusions}

RYGB and MGBP may be more suitable for obesity, but TG and RYGB are recommended for diabetes. Their underlying mechanism may be modulation of ghrelin decrease for TG, ghrelin increase for MGBP and RYGB, and ghrelin/obestatin ratio decrease for SG.

\section{List of Abbreviations}

T2DM: Type 2 diabetes mellitus

RYGB: Roux-en-Y gastric bypass

MGB: Minigastric bypass

SG: $\quad$ Sleeve gastrectomy

BMI: Body mass index

SO: Sham operation

TG: Total gastrectomy

SD: Sprague-Dawley

GK: Goto-Kakisaki.

\section{Conflict of Interests}

The authors have no potential conflict of interests to declare.

\section{Authors' Contribution}

Donglei Zhou and Xun Jiang contributed equally to this work.

\section{References}

[1] K.-H. Yoon, J.-H. Lee, J.-W. Kim et al., "Epidemic obesity and type 2 diabetes in Asia," The Lancet, vol. 368, no. 9548, pp. 1681$1688,2006$.

[2] Organization WH. Fact sheet: obesity and overweight. World Health Organization, 2006.

[3] M. L. Vetter, L. F. Faulconbridge, V. L. Webb, and T. A. Wadden, "Behavioral and pharmacologic therapies for obesity," Nature Reviews Endocrinology, vol. 6, no. 10, pp. 578-588, 2010.

[4] D. Mozaffarian, T. Hao, E. B. Rimm, W. C. Willett, and F. B. $\mathrm{Hu}$, "Changes in diet and lifestyle and long-term weight gain in women and men," The New England Journal of Medicine, vol. 364, no. 25, pp. 2392-2404, 2011.

[5] V. L. Gloy, M. Briel, D. L. Bhatt et al., "Bariatric surgery versus non-surgical treatment for obesity: a systematic review and meta-analysis of randomised controlled trials," British Medical Journal, vol. 347, 2013.

[6] H. Buchwald, Y. Avidor, and E. Braunwald, "Bariatric surgery. A systematic review and meta-analysis," ACC Current Journal Review, vol. 14, no. 1, 13 pages, 2005.

[7] W.-J. Lee, K. Chong, K.-H. Ser et al., "Gastric bypass vs sleeve gastrectomy for type 2 diabetes mellitus: a randomized controlled trial," Archives of Surgery, vol. 146, no. 2, pp. 143-148, 2011.

[8] W.-J. Lee, K.-H. Ser, Y.-C. Lee, J.-J. Tsou, S.-C. Chen, and J.C. Chen, "Laparoscopic Roux-en-Y vs. mini-gastric bypass for the treatment of morbid obesity: a 10-year experience," Obesity Surgery, vol. 22, no. 12, pp. 1827-1834, 2012.

[9] W.-J. Lee, P.-J. Yu, W. Wang, T.-C. Chen, P.-L. Wei, and M.-T. Huang, "Laparoscopic Roux-en-Y versus mini-gastric bypass for the treatment of morbid obesity: a prospective randomized controlled clinical trial," Annals of Surgery, vol. 242, no. 1, pp. 20-28, 2005.

[10] R. Padwal, S. Klarenbach, N. Wiebe et al., "Bariatric surgery: a systematic review and network meta-analysis of randomized trials," Obesity Reviews, vol. 12, no. 8, pp. 602-621, 2011.

[11] J. Korner, W. Inabnet, G. Febres et al., "Prospective study of gut hormone and metabolic changes after adjustable gastric banding and Roux-en-Y gastric bypass," International Journal of Obesity, vol. 33, no. 7, pp. 786-795, 2009.

[12] C. L. Roth, T. Reinehr, G.-H. Schernthaner, H.-P. Kopp, S. Kriwanek, and G. Schernthaner, "Ghrelin and obestatin levels in severely obese women before and after weight loss after Rouxen-Y gastric bypass surgery," Obesity Surgery, vol. 19, no. 1, pp. 29-35, 2009.

[13] F. Carrasco, P. Rojas, A. Csendes et al., "Changes in ghrelin concentrations one year after resective and non-resective gastric bypass: associations with weight loss and energy and macronutrient intakes," Nutrition, vol. 28, no. 7, pp. 757-761, 2012.

[14] A. Bohdjalian, F. B. Langer, S. Shakeri-Leiden Mühler et al., "Sleeve gastrectomy as sole and definitive bariatric procedure: 
5-year results for weight loss and ghrelin," Obesity Surgery, vol. 20, no. 5, pp. 535-540, 2010.

[15] M. S. B. Huda, B. H. Durham, S. P. Wong et al., "Plasma obestatin levels are lower in obese and post-gastrectomy subjects, but do not change in response to a meal," International Journal of Obesity, vol. 32, no. 1, pp. 129-135, 2008.

[16] E. Garcia-Fuentes, L. Garrido-Sanchez, J. M. Garcia-Almeida et al., "Different effect of laparoscopic Roux-en-Y gastric bypass and open biliopancreatic diversion of Scopinaro on serum PYY and ghrelin levels," Obesity Surgery, vol. 18, no. 11, pp. 1424-1429, 2008.

[17] C. Holdstock, B. E. Engström, M. Öhrvall, L. Lind, M. Sundbom, and F. A. Karlsson, "Ghrelin and adipose tissue regulatory peptides: effect of gastric bypass surgery in obese humans," The Journal of Clinical Endocrinology \& Metabolism, vol. 88, no. 7, pp. 3177-3183, 2003.

[18] C. Martins, L. Kjelstrup, I. L. Mostad, and B. Kulseng, "Impact of sustained weight loss achieved through Roux-en-Y gastric bypass or a lifestyle intervention on ghrelin, obestatin, and ghrelin/obestatin ratio in morbidly obese patients," Obesity Surgery, vol. 21, no. 6, pp. 751-758, 2011.

[19] W.-J. Lee, T.-C. Chen, K. Ser et al., "Differential influences of gastric bypass and sleeve gastrectomy on plasma nesfatin-1 and obestatin levels in patients with type 2 diabetes mellitus," Current Pharmaceutical Design, vol. 19, no. 32, pp. 5830-5835, 2013.

[20] A. Rodríguez, S. Becerril, V. Valentí et al., "Short-term effects of sleeve gastrectomy and caloric restriction on blood pressure in diet-induced obese rats," Obesity Surgery, vol. 22, no. 9, pp. 1481-1490, 2012.

[21] A. Hajnal, A. Zharikov, J. E. Polston et al., "Alcohol reward is increased after Roux-en-Y gastric bypass in dietary obese rats with differential effects following ghrelin antagonism," PloS ONE, vol. 7, no. 11, Article ID e49121, 2012.

[22] N. Saeidi, E. Nestoridi, J. Kucharczyk, M. Uygun, M. Yarmush, and N. Stylopoulos, "Sleeve gastrectomy and Roux-en-Y gastric bypass exhibit differential effects on food preferences, nutrient absorption and energy expenditure in obese rats," International Journal of Obesity, vol. 36, pp. 1396-1402, 2012.

[23] F. B. Langer, A. Bohdjalian, S. Shakeri-Leidenmühler, S. F. Schoppmann, J. Zacherl, and G. Prager, "Conversion from sleeve gastrectomy to Roux-en-Y gastric bypass-indications and outcome," Obesity Surgery, vol. 20, no. 7, pp. 835-840, 2010.

[24] R. C. Moon, A. F. Teixeira, M. A. Jawad, and R. J. Rosenthal, "Conversion of failed laparoscopic adjustable gastric banding: sleeve gastrectomy or Roux-en-Y gastric bypass?” Surgery for Obesity and Related Diseases, vol. 9, no. 6, pp. 901-907, 2013.

[25] T. Gautier, T. Sarcher, N. Contival, Y. Le Roux, and A. Alves, "Indications and mid-term results of conversion from sleeve gastrectomy to Roux-en-Y gastric bypass," Obesity Surgery, vol. 23, no. 2, pp. 212-215, 2013.

[26] B. Liedman, I. Bosaeus, I. Hugosson, and L. Lundell, "Longterm beneficial effects of a gastric reservoir on weight control after total gastrectomy: a study of potential mechanisms," British Journal of Surgery, vol. 85, no. 4, pp. 542-547, 1998.

[27] K. Takachi, Y. Doki, O. Ishikawa et al., "Postoperative ghrelin levels and delayed recovery from body weight loss after distal or total gastrectomy," Journal of Surgical Research, vol. 130, no. 1, pp. 1-7, 2006.

[28] T. T. Zittel, J. Glatzle, T. Weimar, S. Kless, H. D. Becker, and E. C. Jehle, "Serotonin receptor blockade increases food intake and body weight after total gastrectomy in rats," Journal of Surgical Research, vol. 106, no. 2, pp. 273-281, 2002.

[29] T. Yoshikawa, N. Hiki, M. Taguri et al., "A phase III trial to evaluate the effect of perioperative nutrition enriched with eicosapentaenoic acid on body weight loss after total gastrectomy for T2-T4a gastric cancer," Japanese Journal of Clinical Oncology, vol. 42, no. 5, pp. 459-462, 2012.

[30] E. Lanzarini, A. Csendes, H. Lembach, J. Molina, L. Gutiérrez, and J. Silva, "Evolution of type 2 diabetes mellitus in non morbid obese gastrectomized patients with Roux en-Y reconstruction: retrospective study," World Journal of Surgery, vol. 34, no. 9, pp. 2098-2102, 2010.

[31] J. W. Kim, J.-H. Cheong, W. J. Hyung, S.-H. Choi, and S. H. Noh, "Outcome after gastrectomy in gastric cancer patients with type 2 diabetes," World Journal of Gastroenterology, vol. 18, no. 1, pp. 49-54, 2012.

[32] A. M. Wren, C. J. Small, H. L. Ward et al., “The novel hypothalamic peptide ghrelin stimulates food intake and growth hormone secretion," Endocrinology, vol. 141, no. 11, pp. 4325-4328, 2000.

[33] T. Shiiya, M. Nakazato, M. Mizuta et al., "Plasma ghrelin levels in lean and obese humans and the effect of glucose on ghrelin secretion," The Journal of Clinical Endocrinology \& Metabolism, vol. 87, no. 1, pp. 240-244, 2002.

[34] J. Tong, R. L. Prigeon, H. W. Davis, M. Bidlingmaier, M. H. Tschöp, and D. D'Alessio, "Physiologic concentrations of exogenously infused ghrelin reduces insulin secretion without affecting insulin sensitivity in healthy humans," The Journal of Clinical Endocrinology \& Metabolism, vol. 98, no. 6, pp. 25362543, 2013.

[35] J. Tong, R. L. Prigeon, H. W. Davis et al., "Ghrelin suppresses glucose-stimulated insulin secretion and deteriorates glucose tolerance in healthy humans," Diabetes, vol. 59, no. 9, pp. 21452151, 2010.

[36] J. V. Zhang, P.-G. Ren, O. Avsian-Kretchmer et al., "Medicine: obestatin, a peptide encoded by the ghrelin gene, opposes ghrelin's effects on food intake," Science, vol. 310, no. 5750, pp. 996-999, 2005.

[37] T. Nakahara, T. Harada, D. Yasuhara et al., "Plasma obestatin concentrations are negatively correlated with body mass index, insulin resistance index, and plasma leptin concentrations in obesity and anorexia nervosa," Biological Psychiatry, vol. 64, no. 3, pp. 252-255, 2008. 


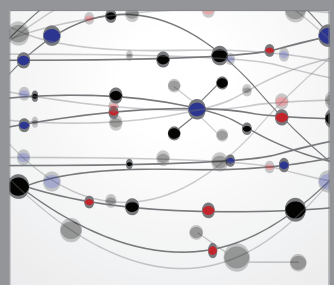

The Scientific World Journal
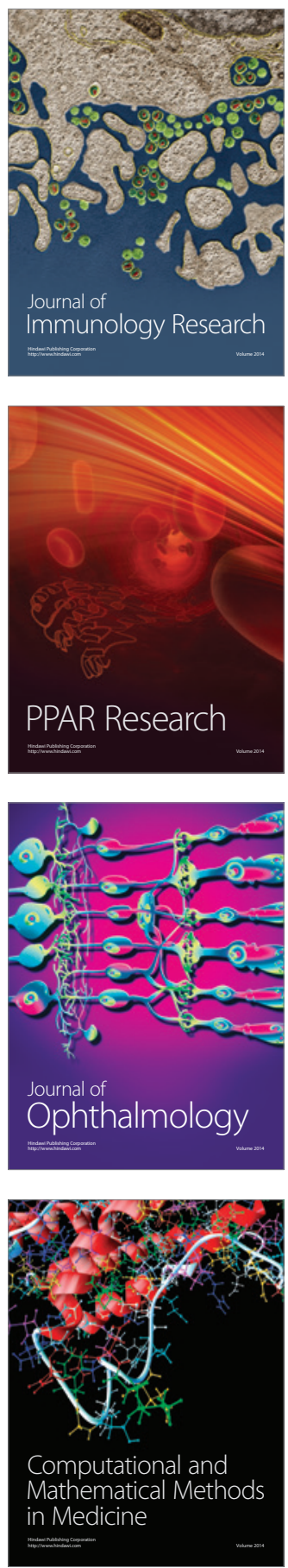

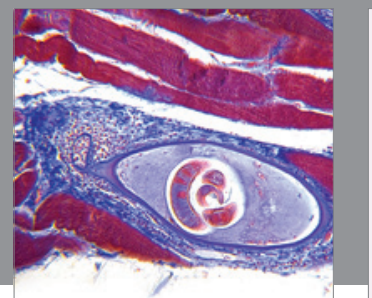

Gastroenterology

Research and Practice
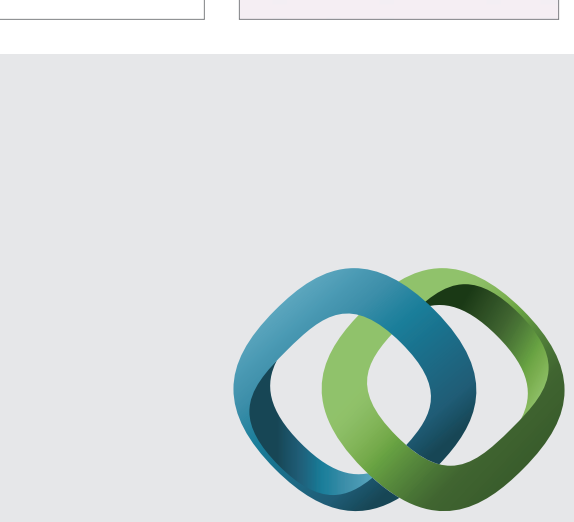

\section{Hindawi}

Submit your manuscripts at

http://www.hindawi.com
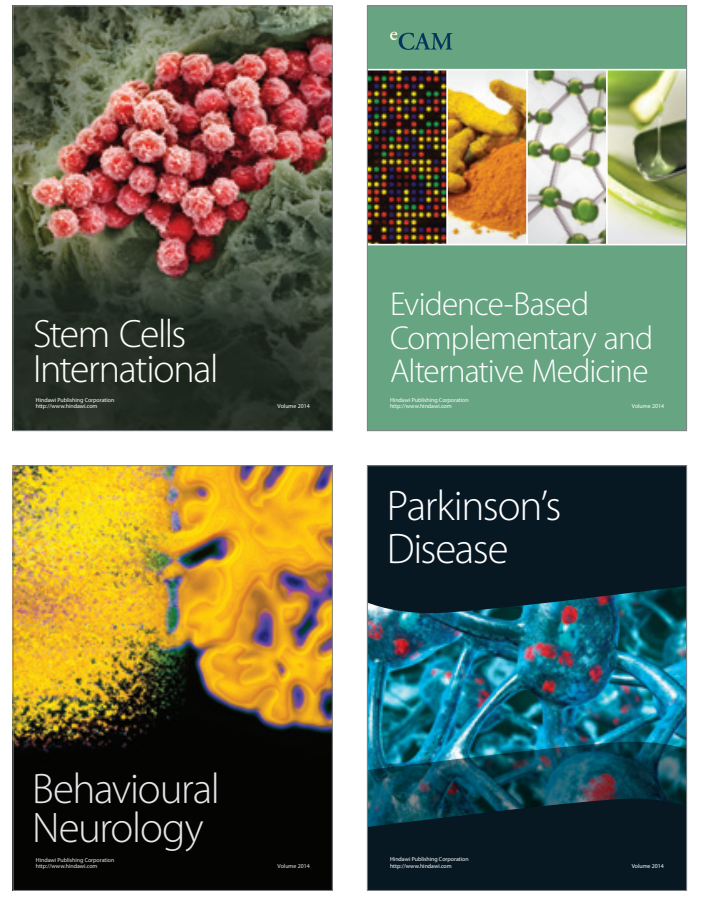
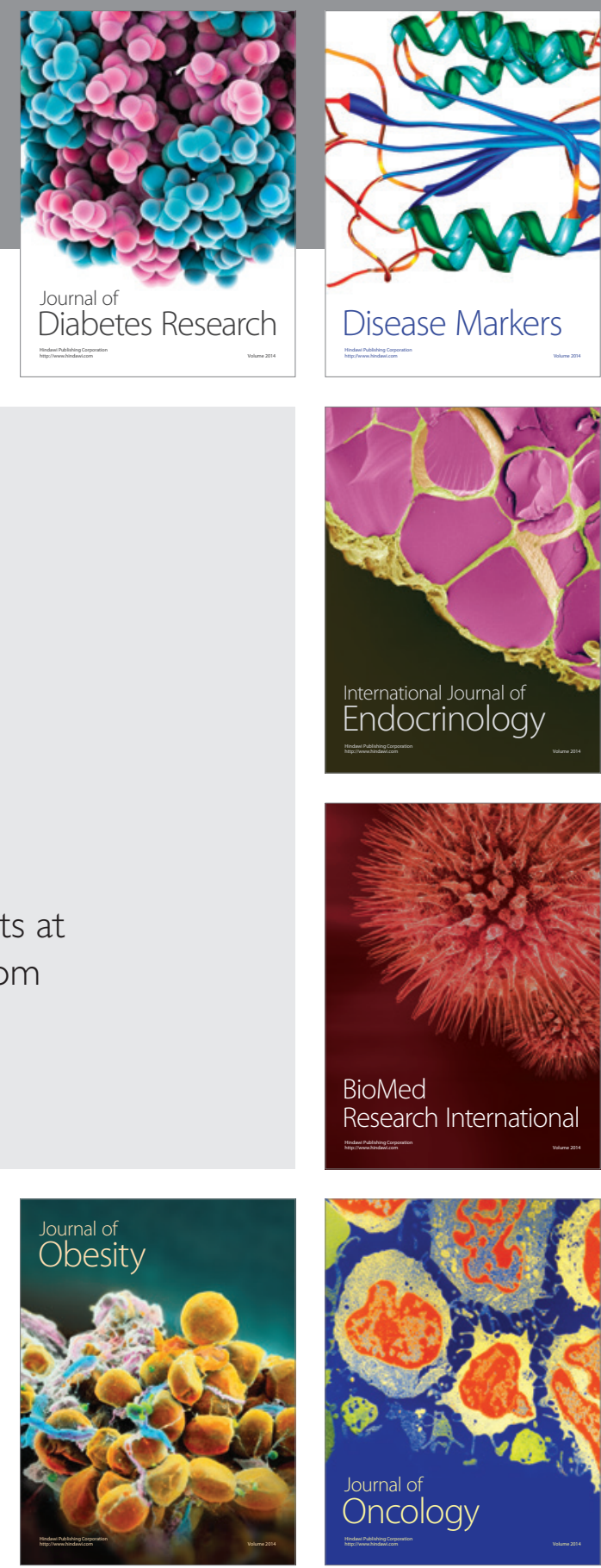

Disease Markers
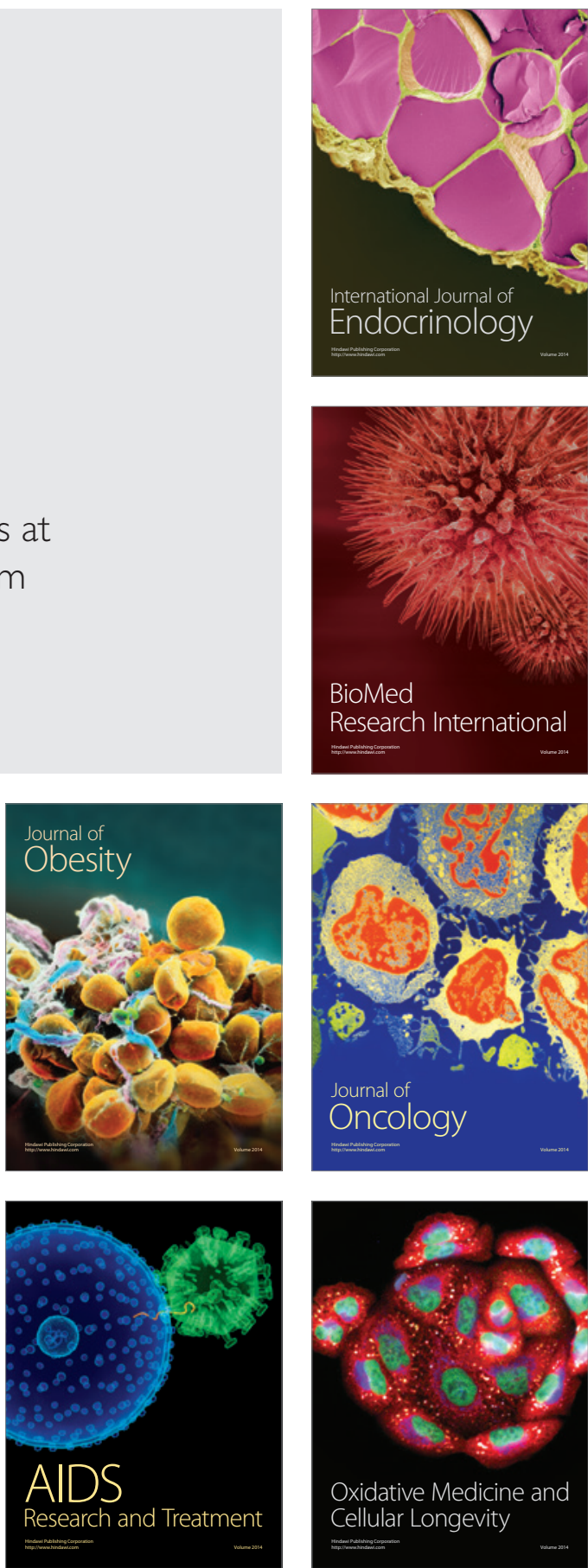